\title{
Improving the Performance Parameters of Microstrip Patch Antenna by using HFSS
}

\author{
Tanvir Singh Buttar, M.Tech. Scholar, Amritsar College Of Engg. \& Tech \\ AMRITSAR, INDIA \\ tanvirbuttar88@gmail.com \\ Narinder Sharma, Associate Professor, Amritsar College Of Engg. \& Tech \\ AMRITSAR, INDIA \\ narinder.acet@gmail.com
}

\begin{abstract}
A high-directivity patch antenna with broadside directivity is appealing, since a constrict beam can be obtained without the need of using an array of antennas. Hence, the solution becomes barer as there is no need for a complicated feeding network. In this senate, this paper presents a novel patch antenna design with high directivity in the broadside direction by using HFSS. The proposed finite element method is employed to design the rectangular microstrip patch antenna. The patch is divided into symmetrical rectangular pieces and has a small overlap area among them. This averts optimized geometries where rectangular pieces have only an infinitesimal connection. Hence, the proposed method is robust for manufacturing. The antenna functions in a higher order mode at $8.40 \mathrm{GHz}, 16.5 \mathrm{GHz}$ and the geometry fits inside a patch of $41 \mathrm{~mm} £ 26 \mathrm{~mm}$ on a substrate with a relative permittivity of 2.1 and a thickness of $1.75 \mathrm{~mm}$ resulting in a gain of 9.94 $\mathrm{dBi}$. The strength of this design is the use of FEM to select the optimized shape and the feeding place instead of a known shape and a fixed feeding place. The antenna has been fabricated and the simulation results are in good agreement with the measurements. These results in a less complicated design of a single high-directivity patch and enhance the gain and improve bandwidth.
\end{abstract}

\section{Indexing terms/Keywords}

Microstrip patch antenna; bandwidth; gain.

\section{Academic Discipline And Sub-Disciplines}

Electronics and Communication, Wireless Technology.

\section{SUBJECT CLASSIFICATION}

Antenna Systems

\section{TYPE (METHOD/APPROACH)}

FEM is used to design the Microstrip patch antenna, Simulation is done by using Ansoft HFSS.

\section{Council for Innovative Research}

Peer Review Research Publishing System

Journal: INTERNATIONAL JOURNAL OF COMPUTERS \& TECHNOLOGY

Vol. 13 , No. 11

www.ijctonline.com , editorijctonline@gmail.com 


\section{INTRODUCTION}

Microstrip patch antennas are the almost mutual form of printed antennas. A microstrip patch antenna is widely utilized in covenant and portable communication devices ascribable to its compact size, dilute profile configurations, accordance and low price. Hence it can be employed in a broad form of applications roaming from mobile communication to satellite, aircraft and other applications [1]. The patch antennas gain can be enhanced by applying multiple patches attached to an array or by coming down the surface wave which can produce ripples in the radiation pattern. Various methods have been aimed to bring down the consequences of surface waves. One approach path proposed is the synthesized substrate that lets down the effectual dielectric constant of the substrate either below or all over the patch [2-3]. Other approach paths are to apply parasitic elements [4-5] or to apply a minimized surface-wave antenna [6]. These structures also have the power to open a band gap, which is a frequency range for which the generation of electromagnetic waves is prevented. By smothering a patch antenna with a square-lattice of small metal pads with grounding vias, also called mushroom-like structure, a significant suppression of surface waves excited in the dielectric substrate has been noticed, which improve the gain of the antenna or efficient radiated ability [9]. Reduce of mutual coupling and co-site interference is other merits of this antenna. The inbuilt drawbacks admit constrict bandwidth and relative prominent size. The requirement of a more minor antenna size much struggle with the desired wide band or multiband applications. Reconfigurable or tunable antennas supply potential results and have appealed significantly research attempts [1-4]. Instead than backing wideband or multiband performances at the same time, accredited antennas are controlled to hop over unlike bands dynamically. Such an antenna would not back all bands simultaneously, merely it grants narrow instant bandwidth that is selectable dynamically.

Since antenna radiation boasts, incubate resonant frequency and radiation patterns are ascertained for the most part by its shapes and size, changing its geometry mechanically appropriate for a non-rational method to reconfigure its radiation attributes. MEMS methods incorporate electrical and mechanical operates in a single component using micro fabrication or micro aching technology, and can be recognized with semiconductor integrated circuit serving [5-7]. Hence, MEMS based reconfigurable antennas had been widely inquired [8-13]. By using printed circuit board (PCB) methods, planar wire antennas in the form of filamentary conductive traces can be constructed with inserted MEMS switches. A MEMS switch changes dynamically the antenna length and enables dual or multiband applications.

Antenna reconfiguration can also be projected electronics switch such as PIN diodes, which are ordinarily applying in modern communications and radar applications. While the diode switch is DC forward biased, it is at an "on" state and is ideally a short circuit; while when the diode is reverse biased, it is at an "off" state and the diode is open-circuited. Placing a PIN diode in among two conducting traces, could assure electronically the efficient antenna physical length. Planar microstrip antennas applying PIN diode switching for reconfiguration had also been investigated [4-8].

Most reconfigurable antennas are grounded on the dynamic control of the antenna physical length. It is potentia to design electronically tunable antennas by adapting the attributes of the material where an antenna occupied. FerriteLoaded microstrip antennas are examples [2], where the antenna features are aligned by the external magnetic fields issued by a dc biased circuit.

In this paper, the functioning of a rectangular microstrip patch antenna has been mended utilizing Finite element method (FEM). The patch antenna is excited by using the microstrip line feed method to raise the antenna gain. The feature of the patch antenna based on embedding a metal boundary in the substrate was studied by [9]. The outcome suggested that the surface waves which spread along the surface of the substrate can be inhibited by the multiple photonic band-gap structure because of its consequences of forbidden band, that it can diversify nearly of electromagnetic waves' energy in the substrate importantly, and that it has frown return loss (S11) compared to the conventional patch antennas and enhanced gain.

\section{ANTENNA CONFIGURATION}

The patch size is fixed to fit in an area of $41 \mathrm{~mm} £ 26 \mathrm{~mm}$. The objective is to maximize the gain within that area. The patch antenna is etched on a thin substrate with a thickness of $1.75 \mathrm{~mm}$. The substrate material is Teflon, which has a relative permittivity of 2.1 and a loss tangent of 0.001 . The antenna is fed by using microstrip feed line method.

\section{Finite Element Method (FEM) Procedure}

In order to generate an electromagnetic field solution, HFSS employs the Finite Element Method (FEM). The FEM divides the full problem space into thousands of smaller regions and represents the field in each sub-region (element) with a local function. In HFSS, the geometric model is automatically divided into a large number of tetrahedral elements, where a ace tetrahedron is a four-sided pyramid. This appeal of tetrahedron is referred to as the finite element mesh. The value of a vector field quantity at points inside each tetrahedron is interpolated from the vertices of the tetrahedron. At each vertex, HFSS stores the components of the field that are tangential to the three edges of the tetrahedron. Moreover, the component of the vector field at the midpoint of selected edges that is tangential to a face and normal to the edge can also be stored. The field within each tetrahedron is interpolated from these nodal values. There is a compromise between the desired level of accuracy and the computation time which depends on the size of the mesh. The accuracy of the solution depends on how small each of the individual elements is. Solutions based on meshes that use a large number of elements are more accurate than solutions based on coarse meshes using relatively few elements. On the other hand, generating a field solution for meshes with a large number of elements requires a significant amount of computing power and memory. Hence, it is desirable to use a mesh that is fine enough to obtain an accurate field solution but not so fine that it overwhelms the available computer memory and processing power or cost too much computation time. 
To produce an optimal mesh, HFSS uses an iterative process in which the mesh is automatically refined in critical regions. First, it gives a solution based on a coarse sign mesh. Then, it rectifies the mesh based on suitable error criteria and generates a new solution. When S-parameters converge within a hoped limit, the reiterative process ends. This limit is defined as Delta Error on the options avail on HFSS. In this simulation, the Delta Error is taken as 0.02 which defines convergence within $2 \%$ limit.

\section{Microstrip Feed Line Technique}

The microstrip antenna can be excited by different techniques. These techniques are classified into two categories: contacting and non-contacting. In non-contacting technique, the electromagnetic field coupling is done to transfer power between radiating patch and microstrip line where as in contacting technique, the RF power is directly fed to the radiating patch by using a connecting element microstrip line. The microstrip line feeding and coaxial probe feeding are the contacting techniques whereas the aperture coupling and proximity coupling are the non-contacting techniques.

In our antenna design we use the microstrip line feeding technique as shown in figure 1.

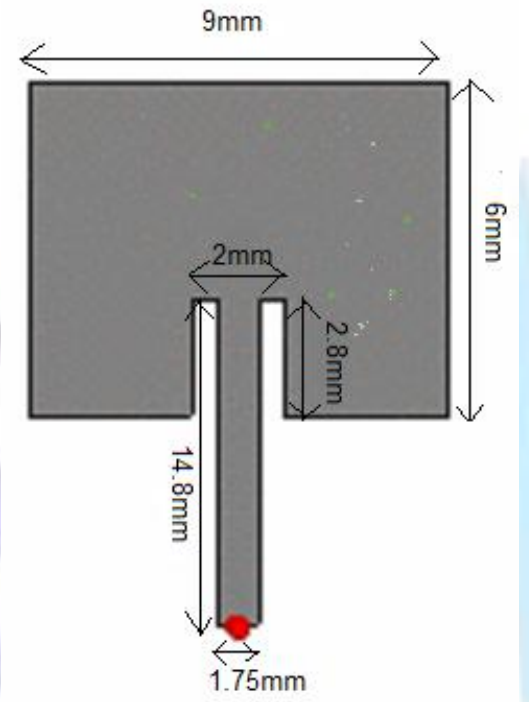

Fig 1. Microstrip feed line of microstrip patch antenna

The above figure depicts the microstrip feed line and its specification are: the cut width $=2 \mathrm{~mm}$, cut depth $=2.8 \mathrm{~mm}$, transmission line or path length $=14.8 \mathrm{~mm}$, width of the feed line $=1.7 \mathrm{~mm}$.

\section{ANALYSIS AND DESIGN \\ Rectangular microstrip patch antenna}

This subsection identifies the patch-antenna configuration that is chose to execute the comparison between using a normal substrate and our proposed microstrip patch antenna. The antenna is depicted in Fig.2. The patches used in our antenna design are cut into symmetrical rectangular shape pieces with Length $=9 \mathrm{~mm}$ and Breath $=6 \mathrm{~mm}$ laid over the dielectric substrate with a relative permittivity of or $=2.1$, which is thick in size with low dielectric constant to achieve the better efficiency. Next, the input impedance of the patch at the edge was determined by placing a length of $50 \Omega$ transmission line at the edge. The sizing of the substrate is $50 \mathrm{~mm} \times 64 \mathrm{~mm}$. The antenna resonates at $8.40 \mathrm{GHz}$ and $16.50 \mathrm{GHz}$.

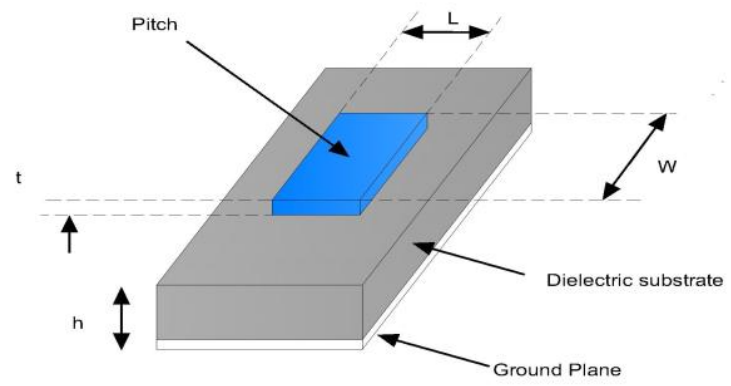

Fig 2: Rectangular patch antenna. 
The maximum gain of the patch antenna is about $9.94 \mathrm{~dB}$. The gain of this antenna is heightening using a FEM, which is depicted in the next subsection.

\section{Patch Antenna Used by FEM}

While the previous subsection has defined the patch-antenna configuration, the design of the FEM substrate is instantly introduced. Figure 3 shows the schematic of the proposed patch antenna surrounded used by structure composed of same patch and grounding vias. The patch is divided into symmetrical rectangular pieces and has a small overlap area among them. This averts optimized geometries where rectangular pieces have only an infinitesimal connection.

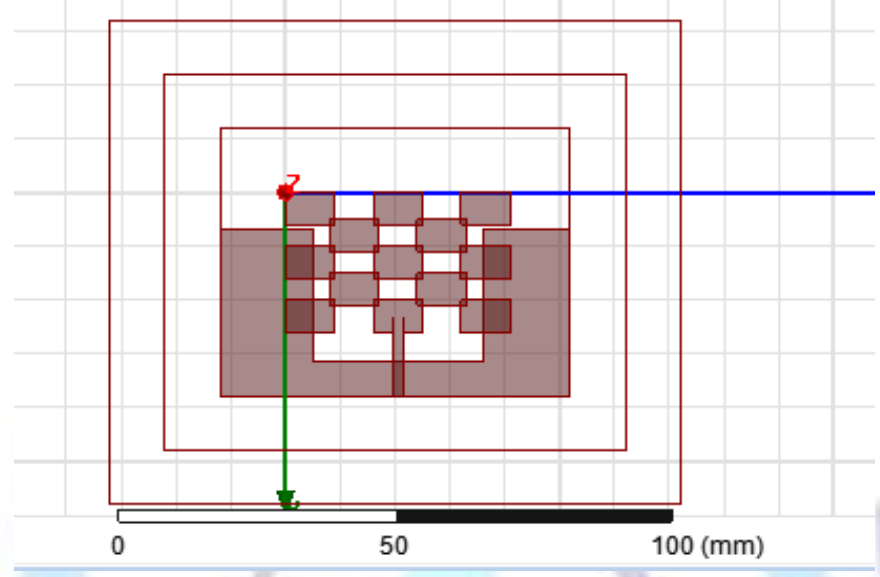

Fig 3: Proposed antenna design using FEM in HFSS.

\section{SIMULATION RESULT}

The proposed antenna has been designed and simulated using Ansoft HFSS software. Results have been analyzed for variation of gain and reflection loss because FEM structures are expected to increase gain by suppression of surface waves. The radiation patterns of the proposed design in $\mathrm{E}$-plane and $\mathrm{H}$ - plane respectively.

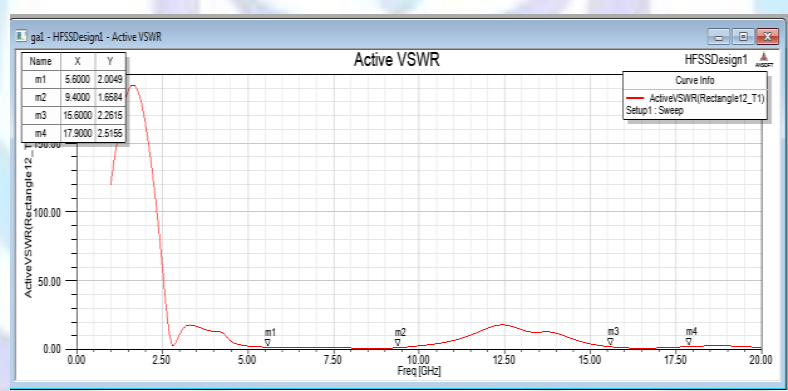

Fig 3: Active VSWR vs. frequency (in $\mathrm{GHz}$ ) for the proposed structure.

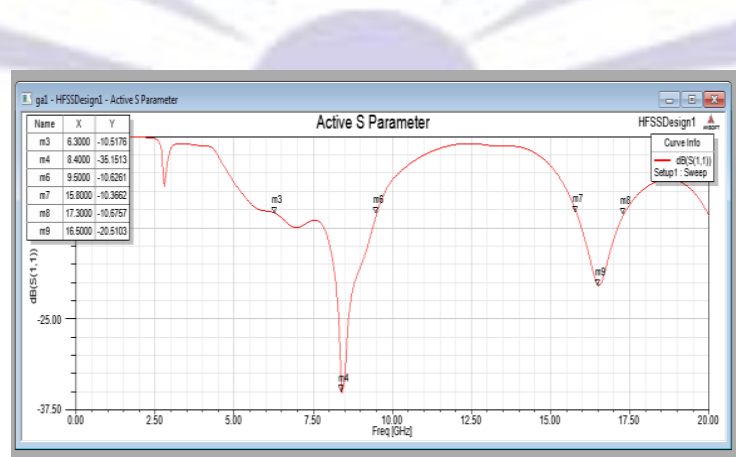

Fig 4: Transmission coefficients (in $\mathrm{dB}$ ) vs. frequency (in $\mathrm{GHz}$ ) for the proposed structure. 


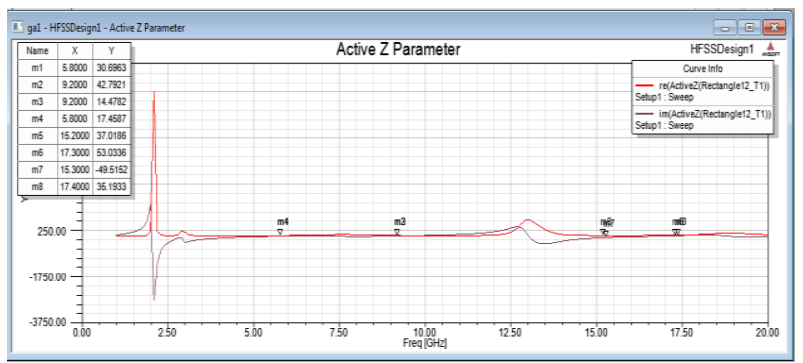

Fig 5: Active $\mathrm{Z}$ parameters vs. frequency (in $\mathrm{GHz}$ ) for the proposed structure.

\section{OBSERVATIONS AND DICUSSION}

Observations concerning the gain, reflection loss and bandwidth using the proposed antenna structure have been talked about and amended in this section

\section{Bandwidth}

This design achieved an impedance bandwidth of $10.14 \%(6.34-17.30 \mathrm{GHz})$ at $-10 \mathrm{~dB}$ return loss. Simulated radiation pattern is nearly omnidirectional. The simulated results show the compatibility of the antenna with the $5.60 \mathrm{GHz}$ ISM band applications. Further enhancement of the antenna performance with improved design is under consideration.

\section{Gain}

Gain has been accounted from the radiation patterns in $E$ and $H$-plane for the patch antenna. Gain experience from proposed antenna structure is found to be $9.94 \mathrm{~dB}$ at $8.40 \mathrm{GHz}$.

\section{Reflection Loss and VSWR}

Reflection loss obtained from our design is $-35 \mathrm{~dB}$ at $8.40 \mathrm{GHz}$ and $-20 \mathrm{~dB}$ at $16.50 \mathrm{GHz}$. It is evident from the reflection loss curves of the antenna structures using FEM and that without FEM that reflection loss has reduced drastically for patch with FEM. This has ensued in a noteworthy improvement in gain. The bandwidth of the constitute FEM-based patch is found to be $112.3 \mathrm{MHz}$.

The obtained value of VSWR at $8.40 \mathrm{GHz}$ is 1.03 and at $16.50 \mathrm{GHz}$ is 1.20 respectively. Ideally, VSWR must lie in the range of $1-2$ which is achieved in our design.

\section{Input Impedence}

The input impedance of the antenna at $8.40 \mathrm{GHz}$ is found to be $50.39-\mathrm{j} 1.71 \Omega$ and at $16.50 \mathrm{GHz}$ is found to be $41+\mathrm{j} 1.01$ $\Omega$ as shown in figure 1,2 . This shows that good impedance matching is achieved.

It is known that the gain of the antenna can be enhanced by contracting any loss of the antenna. Selection of an effective quality (low-loss) substrate will contract the dielectric and conductor losses. Furthermore, surface waves go inside the substrate and reduce the gain. To reduce this surface wave loss, wave propagation has to be blocked inside or reflected outside the substrate to enhance the gain in forward way. The waves are halted or retarded by obstructing their path with a high propagation impedance-air $(\varepsilon r=1)$. This implies that the impedance offered, is maximum for smallest value of $\varepsilon_{r}$ (the smallest value of $\varepsilon_{\mathrm{r}}$ being 1 for air).

\section{CONCLUSION}

In this paper, the micro-strip patch antenna is designed by using a FEM in Ansoft HFSS. The simulated results provide gain, bandwidth, input impedance, VSWR, return loss improvement, which encourages fabricating the structure. The performance parameters can be improved further by using another analysis method like full-wave method. The different feed technique also affects the performance of the antenna.

\section{ACKNOWLEDGMENTS}

The authors like to express their thanks to the department of ECE and the management of Amritsar College Of Engg. \& Tech for their support and encouragement during this work.

\section{REFERENCES}

[1] Bowman, M., Debray, S. K., and Peterson, L. L. 1993. Reasoning about naming systems. .

[2] Ding, W. and Marchionini, G. 1997 A Study on Video Browsing Strategies. Technical Report. University of Maryland at College Park.

[3] Fröhlich, B. and Plate, J. 2000. The cubic mouse: a new device for three-dimensional input. In Proceedings of the SIGCHI Conference on Human Factors in Computing Systems 
[4] Tavel, P. 2007 Modeling and Simulation Design. AK Peters Ltd.

[5] Sannella, M. J. 1994 Constraint Satisfaction and Debugging for Interactive User Interfaces. Doctoral Thesis. UMI Order Number: UMI Order No. GAX95-09398., University of Washington.

[6] Forman, G. 2003. An extensive empirical study of feature selection metrics for text classification. J. Mach. Learn. Res. 3 (Mar. 2003), 1289-1305.

[7] Brown, L. D., Hua, H., and Gao, C. 2003. A widget framework for augmented interaction in SCAPE.

[8] Y.T. Yu, M.F. Lau, "A comparison of MC/DC, MUMCUT and several other coverage criteria for logical decisions", Journal of Systems and Software, 2005, in press.

[9] Spector, A. Z. 1989. Achieving application requirements. In Distributed Systems, S. Mullender

[10] Ravi Kant and D.C.Dhubkarya, "Design \& Analysis of H-Shape Microstrip Patch Antenna", IEEE Transaction in the Global Journal of Research in Engineering, Vol. 10, Issue 6 (Ver 1.0), pp. 26-29, Nov. 2010.

[11] Zavosh and Aberle, "Improving the performance of microstrip-patch antennas" Antennas and Propagation Magazine, IEEE, Vol. 38, Issue No. 4 , pp. 7-12, Aug. 1996.

[12] Panda, A.K. and Sahu, A., "An Investigation of Gain Enhancement of Microstrip Antenna by Using Inhomogeneous Triangular Metamaterial" International(IEEE) Conference on _Computational Communication networks (CICN), pp. 154-157, Gwalior, 7-9 Oct. 2011.

\section{Author' biography with Photo}

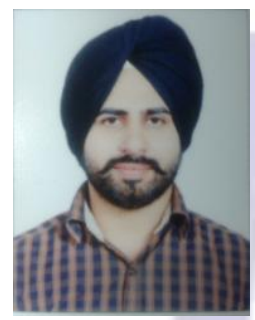

Tanvir Singh Buttar, he is M.Tech (ECE) student at Amritsar college of Engineering \& technology, AMRITSAR. He has earlier completed her B.Tech in ECE from ACET, Amritsar. His area of interestare Antenna \& Wave Propagation, Antenna designing and fabrication, Signal Processing, and optical fiber communication. He is working as an Assistant Professor (ECE Department) in BKSJEC, Amritsar, India.

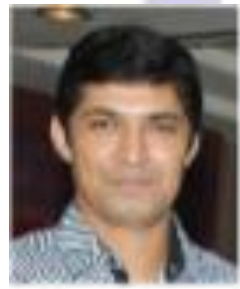

Narinder Sharma, he is working as HOD of EEE at Amritsar College of Engineering and Technology. $\mathrm{He}$ is B.Tech, M.Tech, and Qualified. He has attended many international and National conferences and have Published Paper in national and International Journal. 